\title{
Les réformes institutionnelles du gouvernement Blair : une mise en perspective historique
}

The Blair Government's Constitutional Reforms: an Historical Perspective

\section{Philippe Chassaigne}

\section{OpenEdition}

\section{Journals}

Édition électronique

URL : http://journals.openedition.org/rfcb/694

DOI : $10.4000 /$ rfcb.694

ISSN : 2429-4373

Éditeur

CRECIB - Centre de recherche et d'études en civilisation britannique

\section{Édition imprimée}

Date de publication : 1 février 2002

Pagination : 7-17

ISBN : 2-911580-13-3

ISSN : 0248-9015

\section{Référence électronique}

Philippe Chassaigne, «Les réformes institutionnelles du gouvernement Blair : une mise en perspective historique », Revue Française de Civilisation Britannique [En ligne], XI-3 | 2002, mis en ligne le 21 mars 2016, consulté le 30 avril 2019. URL : http://journals.openedition.org/rfcb/694 ; DOI : 10.4000/ rfcb.694

Ce document a été généré automatiquement le 30 avril 2019.

\section{(c) (i) (3)}

Revue française de civilisation britannique est mis à disposition selon les termes de la licence Creative Commons Attribution - Pas d'Utilisation Commerciale - Pas de Modification 4.0 International. 


\title{
Les réformes institutionnelles du gouvernement Blair : une mise en perspective historique
}

\author{
The Blair Government's Constitutional Reforms: an Historical Perspective
}

\author{
Philippe Chassaigne
}

Le gouvernement de Tony Blair ambitionne, c'est l'évidence même, d'entrer dans l'Histoire comme l'un de ceux qui, au $\mathrm{XX}^{\mathrm{e}}$ siècle, auront fait le plus en matière de transformation institutionnelle du Royaume-Uni. Le défi en la matière, il est vrai, pouvait paraitre facile à relever: au pays de la stabilité politique et de la continuité des structures, le bilan des réformes à l'échelle du siècle écoulé peut être ramené à une poignée de dates: 1911, avec le vote du Parliament Act limitant les compétences législatives de la Chambre des Lords; 1918, avec l'octroi du droit de vote à tous les hommes adultes (à partir de 21 ans) et aux femmes de plus de 30 ans; 1928, et l'alignement de la majorité électorale des femmes sur celle des hommes; 1949, second Parliament Act restreignant encore un peu plus les pouvoirs des Lords et abolissant le vote multiple; 1969, enfin, pour la loi qui ramenait la majorité électorale à 18 ans. En revanche, quelle avalanche de textes votés depuis 1997 ! Devolution en faveur de l'Écosse et du pays de Galles, institution d'un maire et d'une Assemblée élus pour Londres, réforme de la Chambre des Lords consacrant la (quasi) disparition des pairs héréditaires, loi sur la liberté d'information, incorporation de la Convention européenne des Droits de l'homme dans le droit anglais, notamment. Le phénomène est d'autant plus remarquable que les questions institutionnelles n'occupaient pas une place prioritaire dans le manifeste électoral du Labour. Au-delà de la relation des péripéties de la mise en place de ces différents textes, il paraissait nécessaire de replacer un tel travail législatif dans une perspective plus vaste, élargie à l'ensemble du XXe siècle, et d'en apprécier les éléments véritablement novateurs à la lumière de la longue durée. Toutes les propositions, par exemple, n'avaient pas la même portée : si les unes visaient, à l'évidence, à corriger certaines dérives, ou supposées telles, constatées au cours des dix-huit années de gouvernement conservateur, d'autres venaient relancer des projets en suspens depuis 
plus ou moins longtemps. Il conviendra aussi de situer l'entreprise de modernisation politique engagée par Tony Blair par rapport aux questions de fond qui structurent le système de pouvoir britannique, tels que les rapports entre «centre » et " périphérie » (ou pouvoir central et pouvoir local), ou encore la question de la concentration des pouvoirs entre les mains du premier ministre.

\section{Les réformes institutionnelles dans le projet travailliste : une place limitée pour un programme ambitieux}

2 Le programme que le New Labour de Tony Blair présentait aux électeurs britanniques le $1^{\mathrm{er}}$ mai 1997 accordait aux réformes institutionnelles une place certes importante, mais qui était loin d'être essentielle. Elles ne figuraient ainsi pas au nombre des cinq engagements électoraux de base qui servaient de viatique à tous les candidats travaillistes (réduire le nombre des élèves par classes ; punir plus rapidement les délinquants juvéniles ; réformer le service national de santé ; créer 250000 emplois pour les jeunes; ne pas augmenter les impôts). Dans les dix thèmes autour desquels s'articulait, de façon plus substantielle, le manifeste électoral du parti, la nécessité de "nettoyer la vie politique » ("Clean up Politics » tel était l'intitulé du chapitre traitant des réformes institutionnelles) était énoncée en neuvième position, passant avant la volonté de " donner à la Grande-Bretagne un rôle moteur en Europe ", mais arrivant après l'éducation $\left(n^{\circ} 1\right)$, une meilleure répartition des richesses $\left(n^{\circ} 2\right)$, le soutien aux entreprises $\left(n^{\circ} 3\right)$, la réinsertion des chômeurs sur le marché du travail $\left(n^{\circ} 4\right)$, la réforme du NHS $\left(n^{\circ} 5\right)$, la lutte contre la criminalité $\left(n^{\circ} 6\right)$, la défense de la famille $\left(n^{\circ} 7\right)$ et un huitième point aux allures de fourre-tout - «mieux profiter de la vie» ( "get more out of life ») - où coexistaient la protection de l'environnement, l'amélioration des moyens de transport, les arts et la culture... et la réforme de la loterie nationale ! ${ }^{1}$

On peut se demander si cette position relativement subalterne ne reflétait pas l'importance limitée des réformes institutionnelles, au vu des sondages pré-électoraux, dans les préoccupations immédiates des Britanniques. "Nettoyer la vie politique » faisait d'ailleurs directement référence au sleaze, l'avalanche de scandales en tout genre qui avait éclaboussé le gouvernement de John Major tout au long de ses dernières années et contribué à le discréditer aux yeux de l'opinion. De fait, l'une des promesses faites dans le chapitre correspondant était bien de "réforme[r] le financement des partis politiques pour mettre un terme aux scandales $"^{2}$. Toutefois, les quatre pages par lesquelles le New Labour développait ses propositions faisaient apparaitre des projets de bien plus grande ampleur: réformes de la Chambre des Lords et de la Chambre des Communes, modification du mode de scrutin introduisant "une alternative par le biais de la proportionnelle au scrutin majoritaire à un tour», "transparence gouvernementale» ("open government»), transfert de compétences en faveur de l'Écosse et du pays de Galles, réforme du gouvernement local, notamment par l'élection au suffrage universel d'un maire de Londres et d'un conseil municipal, mais aussi par l'institution, en Angleterre, d'assemblées régionales, inclusion de la Convention européenne des Droits de l'homme dans le droit anglais, règlement de la question d'Irlande du Nord. Pareille volonté réformatrice n'était cependant pas sans limite: une phrase venait ainsi rappeler que le parti travailliste «ne prévoyait pas d'abolir la monarchie »3. Partout prévalait la dénonciation des défauts des conservateurs, marqués du péché originel de «s'opposer à l'idée même de 
démocratie » - le manifeste allait même jusqu'à rappeler l'hostilité qui, en son temps, avait été la leur quant au suffrage universel et au vote des femmes - et plusieurs des mesures proposées faisaient nettement figure de réaction à certaines pratiques, institutionnelles ou politiques, qui s'étaient développées entre 1979 et 1997.

\section{Une réaction contre dix-huit années de gouvernement conservateur}

4 La référence la plus immédiate était, bien sûr, celle qui concernait le sleaze, cette succession quasi ininterrompue de scandales mêlant finance et politique - sans compter des questions de moralité privée - qui avait empoisonné le gouvernement Major, de l'affaire Matrix-Churchill en 1992 (ou Arms for Iraq: vente d'armes à l'Irak en dépit de l'embargo), à laquelle le manifeste faisait expressément référence, à Cash for Questions (des députés conservateurs auraient été payés par des groupes d'intérêts pour poser de «bonnes » questions lors des séances de la Chambre des Communes), en 1995-1997. Le fait est que le thème de la corruption tenait une place déterminante dans les préoccupations des Britanniques, comme allait le démontrer le succès de la candidature de l'ancien journaliste de la BBC, Martin Bell, « candidat anti-corruption » auto-proclamé, et premier député indépendant à entrer aux Communes depuis 1945.

Un deuxième point portait sur le mode de scrutin. Les critiques du first past the post system s'étaient accentuées au vu des résultats des législatives de 1983, 1987 et 1992. À chaque fois, le décalage était flagrant entre non seulement le pourcentage des voix obtenu par le parti vainqueur et ses concurrents, mais aussi entre ses différents compétiteurs. En 1983, pour prendre le cas le plus flagrant, les conservateurs, avec $42,4 \%$ des voix, avaient obtenu $53 \%$ des sièges, alors que les travaillistes, avec $27,6 \%$ des suffrages, $33 \%$ des sièges et l'Alliance libéraux/sociaux-démocrates, avec $25,4 \%$ des suffrages, seulement $4 \%$ des sièges. En 1983, 1987 et 1992, avec à chaque fois un pourcentage plus faible des suffrages exprimés, les Tories avaient obtenu des majorités que l'on pouvait qualifier, au pire de confortables (21 sièges en 1992), au mieux d'écrasantes (144 sièges en 1983). À ce système réputé injuste, la représentation proportionnelle apparaissait comme un gage d'équité démocratique; des associations comme le Voting Reform Group, l'Electoral Reform Society, ou Democratic Audit en réclamaient l'instauration, sous une forme ou sous une autre, et le parti travailliste s'était engagé dès 1996, en accord avec les libérauxdémocrates, à lancer une réflexion à ce sujet en cas de retour au pouvoir (création du Joint Consultative Committee on Constitutional Reform). On parlait beaucoup de l'Additional Member System, parfois rebaptisé Additional Vote, combinant, à l'allemande, scrutins majoritaire et proportionnel ; effectuées selon ce système, les législatives de 1992, par exemple, auraient vu les conservateurs obtenir 316 députés au lieu de 336, les travaillistes 240 au lieu de 271, mais les libéraux-démocrates auraient eu 74 élus, et non pas seulement $20^{4}$. Le parti de John Major serait resté le plus nombreux des Communes, mais aurait manqué de 10 sièges la majorité absolue ; pareille configuration était perçue comme plus proche des volontés exprimées par les électeurs dans les urnes.

6 Le point principal, cependant, se rapportait aux accusations portées de façon répétée contre les conservateurs d'avoir pratiqué un système de gouvernement de plus en plus autoritaire, et ce, à tous les niveaux. La multiplication des quangos (quasi-autonomous nongovernmental organisations, conseils ou commissions créés par décision ministérielle) était 
vue comme une façon de transférer des pouvoirs de corps élus vers des corps non-élus et, surtout, comme permettant au clientélisme de régner en maitre dans la nomination des membres ; c'était cet aspect-là que dénonçait en 1995 le rapport de la commission Nolan sur les règles de bonne conduite dans la vie publique (Nolan Committee on Standards in Public Life). De fait, l'estimation du nombre des quangos variait de 1200 , selon le gouvernement conservateur, à 7700 selon des enquêtes indépendantes. Par ailleurs, dans tout système parlementaire, la question des rapports entre exécutif et législatif revêt une importance capitale; le procès ici instruit était celui de la subordination des Communes au rang d'une "assemblée sans pouvoir » ${ }^{5}$. L'image était en effet profondément ancrée dans l'opinion d'une dame de fer ayant exercé, du fait des confortables majorités dont elle disposait au cours de chacune de ses trois législatures et de son autorité sur le parti conservateur, une domination sans partage sur la Chambre basse du Parlement. Alors que, sous les gouvernements Wilson et Callaghan (1974-1979), les députés avaient pu bénéficier d'une réelle marge de manœuvre sur la politique gouvernementale ${ }^{6}$, cette capacité d'intervention aurait disparu par la suite, conduisant à une centralisation accrue du pouvoir entre les mains du gouvernement. Ce dernier aurait, en outre, profité de cette absence de contrôle par le législatif pour pratiquer de nombreux abus, justifiés, au moins en rhétorique, par les intérêts supérieurs de l'État.

7 Ces affirmations n'étaient pas totalement exactes : les gouvernements Thatcher et Major avaient ainsi dû, à plusieurs reprises, retirer des textes face à la fronde de leurs députés de base ${ }^{7}$, mais ces réalités étaient largement obérées par une affaire comme Spycatcher, dans laquelle le gouvernement Thatcher avait voulu empêcher par voie judiciaire le Guardian, l'Observer et le Sunday Times de publier des extraits d'un livre de mémoires d'un ancien agent des services secrets britanniques ${ }^{8}$. La publication, sous le mandat de John Major, d'un Livre blanc sur la question (juillet 1993) ${ }^{9}$, selon les termes duquel chaque ministère devrait, au nom de la «transparence» (open government) publier les informations statistiques ou autres données factuelles sur lesquelles se fondaient ses décisions, ne pouvait que décevoir ceux qui réclamaient une véritable loi sur la liberté de l'information, d'autant que tout ce qui relevait des questions de sécurité était exclu desdites dispositions.

Cette question du droit d'accès à l'information officielle renvoyait elle-même à la situation du Royaume-Uni, pays dépourvu d'une Déclaration des droits. L'engagement du Labour en faveur de l'incorporation de la Charte européenne des Droits de l'homme ${ }^{10}$ venait répondre aux revendications du mouvement Charter 88 , fondé en 1988, à l'occasion du tricentenaire de la Glorieuse révolution et du Bill of Rights ${ }^{11}$. Regroupant intellectuels, universitaires et figures politiques de gauche (dont Lord Scarman, auteur du célèbre rapport de 1981 sur les émeutes de Brixton), il militait en faveur d'une véritable déclaration des droits de la personne.

9 Un dernier élément concernait le gouvernement local: pour les critiques du gouvernement Thatcher, celui-ci l'avait "émasculé»" notamment avec le Local Government Act de 1985, qui avait aboli le Greater London Council et les six Metropolitan County Councils institués par Edward Heath en 1972. En outre, l'administration centrale avait progressivement réduit les pouvoirs financiers des autorités locales par le biais $\mathrm{du}$ plafonnement des impôts locaux (rate capping), avant de mettre en place la réforme de la poll tax ${ }^{13}$ dont l'impopularité devait, on le sait, coûter son poste à Margaret Thatcher (novembre 1990). John Major avait rapporté cette mesure et rétabli peu ou prou l'ancien système, en l'espèce, la council tax, tout en conservant le principe du plafonnement. La 
mise en application du programme néo-libéral de Margaret Thatcher était passée par une centralisation accrue, dans la mesure où les autorités locales, souvent entre les mains des travaillistes, pratiquaient l'obstruction systématique contre les mesures décidées à Londres. Le manifeste travailliste entendait revenir sur ces pratiques autoritaires en limitant l'intervention du gouvernement central au «cadre de base » et rendant davantage d'initiative aux autorités locales pour "encourager le bien-être économique, social et environnemental de leur circonscription $»^{14}$.

En matière de vie politique comme ailleurs, les travaillistes s'engageaient donc à faire souffler un vent nouveau après dix-huit années de domination tory et ils se posaient en défenseurs de la morale et du renouveau de la vie démocratique. Toutefois, certaines de leurs propositions allaient plus loin et ressortissaient à des débats anciens de la démocratie britannique.

\section{La reprise des points longtemps en suspens}

11 Le Labour, dans l'opposition depuis dix-huit ans, ne pouvait anticiper le raz-de-marée électoral qui allait le ramener aux affaires. Toutefois, pratiquement assuré, au vu des enquêtes d'opinion, de sa victoire, il pouvait se permettre d'inclure dans son programme des propositions plus hardies, destinées à faire aboutir des réformes enterrées depuis longtemps. C'était le cas, notamment, de la devolution, mais aussi de la réforme de la Chambre des Lords.

La question de la devolution rappelait aux travaillistes les difficultés qui avaient marqué leur dernier passage aux affaires en 1974-1979. Les résultats des deux élections de février et octobre 1974 avaient montré qu'il fallait désormais compter avec les partis nationalistes écossais et gallois ${ }^{15}$. C'est pourquoi le gouvernement Wilson avait présenté, en novembre 1975, dans le Livre blanc Our Changing Democracy, les grandes lignes d'une réforme institutionnelle qui accordait à l'Écosse et au pays de Galles des assemblées régionales autonomes. Le successeur de Wilson, James Callaghan, éprouva cependant les plus grandes difficultés à obtenir l'assentiment des Communes : en juin 1977, il dut retirer un premier Scotland and Wales Bill, qui légiférait de façon globale pour les deux nations celtes; deux textes différents, l'un pour l'Écosse (Scotland Bill), l'autre pour le pays de Galles (Wales Bill) furent finalement adoptés en février 1978, non sans que les Communes eussent au préalable rejeté la disposition qui introduisait le scrutin proportionnel pour les élections à la future Assemblée écossaise. Restait, en vertu de l'amendement introduit par les députés au cours des débats, à soumettre les textes à référendum aux populations concernées. La consultation, organisée le $1^{\text {er }}$ mars 1979, se conclut, on le sait, par le rejet de chacun des projets : la décision était sans appel au pays de Galles ( $79,7 \%$ de «non »), moins nette en Écosse ( $51,5 \%$ de « oui », mais, compte tenu du taux de l'abstention, ceuxci ne représentaient qu'à peine $33 \%$ des inscrits, alors qu'une majorité qualifiée de $40 \%$ était requise) ; le résultat n'en était pas moins incontestable.

Revenus aux affaires au lendemain des législatives d'avril 1979, les conservateurs enterrèrent la réforme et il ne fut plus question, à Whitehall, de devolution au cours des dix-huit années suivantes ${ }^{16}$. Comme l'écrit Andrew Gamble,

[Dans l'optique conservatrice] le meilleur moyen de défendre l'État unitaire n'est pas de procéder à une décentralisation des pouvoirs en faveur d'assemblées régionales, mais d'utiliser les pouvoirs du gouvernement central pour créer un 
espace politique dans lequel les besoins respectifs de l'Écosse et du pays de Galles peuvent être pris en compte. ${ }^{17}$ le Scottish National Party progressait aux élections, tant locales que législatives (il doubla sa part des suffrages entre 1979 et 1997, passant de $11 \%$ à $22 \%$ ) et où, en même temps, l'idée d'une nécessaire décentralisation du pouvoir faisait son chemin dans la population. En outre, les Écossais supportaient de plus en plus difficilement d'être administrés par un gouvernement conservateur, eux qui élisaient de moins en moins de députés tory : 22 en 1979 (sur les 72 élus écossais), 21 en 1983, 10 en 1987, 11 en 1992. En 1990 et en 1995, la Convention constitutionnelle écossaise - un forum de discussion, largement dominé par les travaillistes, mais, même si les conservateurs et les nationalistes boudèrent ses réunions, comprenant aussi des libéraux-démocrates, des Verts, des représentants des Églises, des syndicats - publia deux « rapports au peuple écossais » demandant la mise en place d'un Parlement national. Au pays de Galles, travaillistes, libéraux-démocrates et nationalistes unirent leurs forces pour lancer une Campaign for a Welsh Assembly (1987), qui devint, en 1993, The Parliament for Wales Campaign. Toujours combattue par les conservateurs, l'idée de devolution, pour l'Écosse comme pour le pays de Galles, fut ensuite intégrée aux programmes des partis travailliste et libéral-démocrate dans la perspective des législatives de 1997. différaient en définitive assez peu de ceux qui avaient été proposés aux électeurs près de vingt ans plus tôt. Il s'agissait avant tout de questions de terminologie (ainsi, la Scottish Assembly de 1979 est devenue un "parlement ", et le First Secretary un First Minister; en revanche, les termes d'« assemblée » et de « secrétaire » étaient conservés pour le pays de Galles) ou de points techniques, tels le nombre de membres des assemblées ou le mode de scrutin. On relevait toutefois deux exceptions, notables, pour l'Écosse : d'une part, au lieu d'établir la liste exhaustive des pouvoirs concédés à son Parlement, ce qui était le cas en 1978, le nouveau texte se contentait de dresser la liste des prérogatives retenues par Westminster, tout le reste étant implicitement dévolu - au sens strict - à Édimbourg ; d'autre part, le Parlement écossais se voyait doté des pouvoirs fiscaux qui lui avaient été refusés en $1978^{18}$. En revanche, le degré de dévolution, moindre pour le pays de Galles que pour l'Écosse, ou les pouvoirs concédés à l'une et l'autre assemblée, ne différaient guère. En gros, il s'agissait de tirer parti d'un contexte politique, cette fois extrêmement favorable, pour relancer le processus qui avait achoppé en 1979, en l'amendant çà et là en fonction des développements intervenus au cours des deux décennies écoulées.

La question de l'Irlande du Nord est bien sûr inséparable de celle de la devolution pour les deux autres pays de la Celtic fringe, l'Écosse et le pays de Galles. Du rétablissement de l'administration directe de la province par Londres en 1972 au milieu des années 1980, la situation n'avait pour ainsi dire pas évolué sur le fond. L'accord anglo-irlandais de 1985 avait marqué le début d'une nouvelle phase, dans laquelle s'inscrivaient, au début du mandat de John Major, la déclaration de Downing Street du 15 décembre 1993, le cessezle-feu décidé par l'IRA puis les unionistes en 1994, ou le Joint Framework Document du 22 février 1995. Cependant, ces avancées s'étaient ensuite enlisées devant, d'une part, le refus de l'IRA de s'engager dans le processus de désarmement qui aurait attesté de sa volonté de renoncer définitivement au terrorisme, et, d'autre part, la nécessité pour John Major de se concilier les treize députés unionistes présents à Westminster afin de conserver une majorité aux Communes. Les négociations reprirent dès juillet 1997, 
débouchant sur l'accord de paix du vendredi saint (Good Friday Agreement) du 10 avril 1998, les élections à une nouvelle Assemblée (la première depuis la suspension du Stormont en 1972) au mois de juin suivant, et, finalement, son installation en décembre 1999.

La réforme de la Chambre des Lords constituait le second grand pan des réformes institutionnelles ouvert depuis longtemps, et jamais refermé. En effet, c'était le gouvernement libéral d'Asquith qui, plus de quatre-vingts ans plus tôt, avait procédé à la première réduction des pouvoirs législatifs de la Chambre haute, au terme de la crise constitutionnelle de 1909-1911. Celle-ci avait débuté, on le sait, avec le rejet par les Lords, en novembre 1909, du «budget du peuple», présenté par le Chancelier de l'Échiquier, David Lloyd George, qui comportait une forte hausse de la fiscalité pour les plus hauts revenus, destinée à financer tout un train de mesures sociales. Le débat qui s'ouvrit alors portait sur une question simple: une chambre non élue pouvait-elle aller contre une décision des Communes, dépositaires de la volonté populaire (il convient de rappeler toutefois que cela était, d'un strict point de vue institutionnel, son droit le plus absolu)? La crise ainsi ouverte se dénoua, deux élections législatives anticipées plus tard (janvier et décembre 1910), par l'adoption, en août 1911, d'un Parliament Act qui privait les Lords de tout contrôle sur les textes à caractère fiscal ou financier et ne leur laissait sur les autres projets de loi qu'un droit de veto suspensif d'une durée maximale de deux ans. Toutefois, la réforme n'était conçue que comme partielle : le texte même de la loi incluait, dans ses considérants, l'annonce de l'intention de "substituer à la Chambre des Lords telle qu'elle existe aujourd'hui une Seconde Chambre, reposant sur un principe populaire et non plus héréditaire", tout en ajoutant aussitôt qu' "une telle transformation ne peut être mise immédiatement en application». De fait, plus aucune réforme de fond ne fut envisagée ensuite. Le Parliament Act de 1949, voté à l'initiative du gouvernement travailliste de Clement Attlee, se contentait de ramener à un an la durée du veto suspensif concédé aux Lords par le texte de 1911.

18 C'est cette partie-là de la réforme annoncée en 1911 que le gouvernement Blair s'attacha à mettre en œuvre, en novembre 1999, avec l'éviction des Lords héréditaires - même tempérée par une clause transitoire permettant à 92 d'entre eux, élus par leurs collègues au scrutin secret, de demeurer en place - remplacés, bien entendu, par des pairs à vie, dont le nombre va croissant (plus de 200 nominations depuis mai 1997, soit plus que n'en a nommés Margaret Thatcher pendant le même laps de temps). Toutefois, au vu du flou des projets de réforme ultérieurs, la chambre de transition mise ainsi en place a peut-être des chances de durer aussi longtemps que celle issue de la réforme de 1911...

\section{Les enjeux permanents}

Une vue d'ensemble des réformes institutionnelles se doit d'aller au-delà de l'énonciation d'une typologie binaire entre mesures de nature circonstancielle et réouverture de chantiers délaissés depuis trop longtemps. Une fois menées à leur terme, ces réformes auront introduit des changements structurels importants dans le système de pouvoir britannique et ce, dans trois domaines principaux, qui se trouvent avoir fourni la matière à des débats quasi incessants depuis au moins un demi-siècle : les rapports entre pouvoir central et autorités locales, ceux entre gouvernement et Parlement, et la question du rôle et des pouvoirs du premier ministre. 
20 L'opposition entre «centralisateurs» et «localistes» est, en Grande-Bretagne, pratiquement aussi ancienne que les institutions locales elles-mêmes ${ }^{19}$; depuis 1972, et la réforme d'ensemble des collectivités locales engagée par le gouvernement d'Edward Heath, l'équilibre qui pouvait exister précédemment entre les deux niveaux de pouvoir s'est rompu au bénéfice du « centre » et la tendance n'a fait que s'accentuer après 1979 (cf. supra). En 1997, toutefois, le programme travailliste ne proposait pas de revenir simplement au statu quo ante : les résolutions qu'il annonçait devaient en fait engager une nouvelle phase dans l'histoire des relations entre les deux pôles du système de pouvoir britannique.

Les mesures de transferts de responsabilités décidées en faveur de l'Écosse et du pays de Galles permettent au Royaume-Uni de se rapprocher du système de décentralisation administrative en vigueur dans des États européens tels que l'Espagne, la Belgique ou l'Allemagne. L'éventualité, pour les nationalistes écossais, de se servir de l'autonomie comme d'un marche-pied pour accéder à l'indépendance, demeure en grande partie hypothétique ${ }^{20}$; quant au pays de Galles, on peut dire que la question ne se pose pas. En revanche, c'est la notion même d'un royaume « uni » qui se trouve mise à mal par ces transferts : si « union » n'a jamais signifié « unification » (au moins pour l'Écosse, à qui le traité de 1707 laissait son Église, son droit et son système éducatif), il n'empêche que cette union paraissait bien, aux yeux des Anglais, équivaloir à une extension pure et simple de leur pays. Pareille situation n'est plus de mise aujourd'hui. En Angleterre stricto sensu également, les rapports entre gouvernement central et gouvernement local sont également susceptibles d'être modifiés à terme, si l'on assiste à la mise en place du volet anglais du processus de dévolution, jusqu'alors limité à la seule mise en place d'une assemblée («Autorité stratégique») et d'un maire élus pour Londres. Le manifeste travailliste de 1997 évoquait la possibilité d'instaurer «en son temps» («in time») des gouvernements régionaux élus au suffrage direct ${ }^{21}$, à la suite de référendums locaux auprès des populations concernées. La nature vague de cet engagement explique que rien n'ait été entrepris en ce sens au cours du premier mandat de Tony Blair. La mise en place de huit Agences de développement régional (Regional Development Agencies, ou RDAs) constitue le seul point à l'actif du gouvernement en la matière. Ces RDAs, dirigées par un conseil aux membres nommés par le gouvernement, ont pour mission le développement économique régional, par la coordination des activités des autorités locales de plein droit et des agences régionales héritées des gouvernements précédents (tels les Government Offices in Regions, créés par John Major), et les acteurs du privé. Ils n'ont aucune légitimité démocratique et leurs pouvoirs sont, au total, fort restreints. Le projet d'organiser un référendum dans la seule Angleterre comme préalable à une véritable régionalisation est sans doute reporté au deuxième mandat de Tony Blair ; il n'empêche qu'il s'agira alors d'une évolution significative dans les rapports entre « centre » et " périphérie ».

Une restriction s'impose toutefois, afin de nuancer ce tableau d'un gouvernement prêt à écouter les "localistes » après deux décennies de centralisation à tout crin. En effet, la décentralisation institutionnelle, indéniable, est contrebalancée par une volonté non moins réelle du premier ministre de s'assurer un contrôle direct des institutions mises en place, par le biais du parti. On l'a vu lors de l'élection en février 1999 du chef du parti travailliste gallois (qui était promis, dans le cas de la victoire probable du Labour aux élections régionales, à devenir le First Secretary de la principauté), ou de la longue « querelle de l'investiture » travailliste pour la mairie de Londres en 1999-2000, lorsqu'ont 
été imposés les candidats de l'appareil du parti (Alun Michael et Frank Dobson respectivement) au détriment de ceux qui avaient les faveurs de la base.

On ne saurait donc dire que les pouvoirs de l'exécutif ont reculé, face à ceux d'un législatif dépositaire de l'expression de la volonté populaire. Au contraire, certaines des nouvelles dispositions constitutionnelles vont encore l'accroître. C'est le cas, par exemple, de l'incorporation de la Convention européenne des Droits de l'homme dans le droit britannique : en cas de «déclaration d'incompatibilité » entre une loi nationale et ladite Convention, prononcée par un tribunal britannique, le gouvernement aura le droit de court-circuiter le Parlement pour amender le texte déclaré défectueux par une simple mesure réglementaire. C'en est donc fini du principe, établi au lendemain de la Glorieuse révolution, de la suprématie du Parlement. De même, les pouvoirs du premier ministre, incarnation même du gouvernement central, ne sortent guère amoindris de la nouvelle donne institutionnelle. En l'absence de transformation réelle de la Chambre des Lords en une seconde Chambre, démocratiquement désignée, disposant d'un droit de regard sur le travail législatif des Communes, il exerce un contrôle total sur la politique britannique. L'évolution de fond, enregistrée depuis au moins un demi-siècle, en direction d'un renforcement des pouvoirs du chef du gouvernement ne s'est donc pas arrêtée, et la comparaison avec un « monarque élu $»^{22}$ (au sens étymologique de «pouvoir d'un seul») conserve toute sa pertinence. Les accusations d'autoritarisme, fréquemment adressées à l'encontre de Tony Blair, ne peuvent que paraître fondées, au vu du rôle de simple chambre d'enregistrement auquel est ramené le Cabinet, réuni de moins en moins fréquemment et pour des séances de plus en plus courtes, ou de l'importance revêtue par les conseillers extra-ministériels dans l'élaboration de la politique gouvernementale. Le temps où le premier ministre britannique faisait figure de primus inter pares, de chef d'orchestre du Cabinet, est bel et bien révolu et le leader travailliste, qui devrait pourtant être idéologiquement attaché au principe de la forme collective de gouvernement, ne paraît guère pressé d'y revenir.

Le domaine institutionnel est donc bien celui dans lequel le gouvernement de Tony Blair présente non seulement le bilan le plus impressionnant, mais aussi celui où il s'est écarté le plus radicalement de l'héritage conservateur. Peut-être était-ce parce que, en la matière, sa reconduction au 10 Downing Street paraissait pratiquement acquise. Il suffit, pour cela, de considérer les taux de participation, très bas, aux élections locales. Le paysage institutionnel qui sortira de ces réformes, encore inachevées, sera sans doute plus démocratique, avec une souveraineté populaire plus fréquemment consultée. On n'en note pas moins la persistance, fût-ce par des voies détournées, des pratiques centralisatrices qui ont prévalu depuis trois décennies. Il est difficile d'aller à l'encontre des réflexes traditionnels.

\section{NOTES}

1. New Labour. Because Britain Deserves Better (dorénavant: BBDB), Londres : Labour Party, 1997, p. 5 . 
2. Ibid., p. 33.

3. Ibid., p. 33.

4. Chiffres cités par David M. FARRELL, "La réforme hésitante du système électoral de la Chambre des Communes", Pouvoirs, n 93, "Le Royaume-Uni de Tony Blair", avril 2000.

5. Philip NORTON, "Le Parlement", in Jacques LERUEZ (dir.), La Grande-Bretagne à la fin du XXe siècle , Paris : Documentation française, 1994, p. 51.

6. Du fait de l'étroitesse de la majorité travailliste issue du scrutin d'octobre 1974 ; cf. Philip NORTON, Dissension in the House of Commons 1974-1979, Oxford : Oxford University Press, 1980.

7. On connaît, bien sûr, le cas du Shops Bill (Sunday Trading) de 1986, qui devait autoriser l'ouverture des magasins le dimanche; il y en eut une demi-douzaine d'autres (on en trouvera la liste dans D. et G. BUTLER, Twentieth Century British Political Facts, Basingstoke : Macmillan, 2000).

8. On se reportera à Jean-Claude SERGEANT, La Grande-Bretagne de Margaret Thatcher, Paris : PUF, 1993, p. 166.

9. Open Government, Londres : HMSO, 1993.

10. BBDB, p. 35 .

11. Bien que constituant l'une des pierres angulaires des libertés anglaises, le Bill of Rights n'est pas, rappelons-le, une déclaration des droits de l'individu, mais d'abord de ceux du Parlement par rapport à la Couronne.

12. Pour reprendre l'expression de Robin OAKLEY, in "Thatcher Dominates Shifting Landscape", The Times, 28 décembre 1989.

13. Ou "charge communautaire »: les impôts locaux traditionnels, pesant sur la propriété, étaient remplacés par une taxe par tête, à laquelle tous les adultes étaient assujettis.

14. BBDB, p. 34.

15. Le Scottish National Party avait obtenu successivement 7 et 11 élus. Le Plaid Cymru gallois, 2 puis 3 sièges.

16. Pour l'Écosse, on trouvera l'étude la plus claire de cette période dans Jacques LERUEZ, L'Écosse. Vieille nation, jeune État, Crozon : Armeline, 2000.

17. Andrew GAMBLE, “The National Question”, in Dennis KAVANAGH \& Anthony SELDON (eds.), The Major Effect, Londres : Macmillan, 1994, p. 390.

18. Le Parlement écossais pouvait modifier, dans la limite de $3 \%$, le taux de l'impôt sur le revenu.

19. Une synthèse sur ce point dans Monica CHARLOT, Le Pouvoir politique en Grande-Bretagne, Paris : PUF, 1998, notamment pp. 361-365.

20. Cf. J. LERUEZ, op. cit., pp. 311-315.

21. BBDB, p. 34.

22. Pour reprendre le titre bien connu de l'ouvrage de F.W.G. BENEMY, The Elected Monarch, Londres : Harvey, 1965.

\section{RÉSUMÉS}

Replacées dans une perspective plus vaste, les réformes institutionnelles réalisées par Tony Blair au cours de son premier mandat (1997-2001) semblent avoir été dictées tant par une volonté de remédier à certaines "dérives » enregistrées au cours des dix-huit années de gouvernement conservateur que par le souci de procéder à une modernisation en profondeur de l'architecture institutionnelle du Royaume-Uni. Toutefois, certaines tendances de fond, enregistrées depuis 
plusieurs décennies, telle la centralisation, ou la concentration accrue du pouvoir entre les mains du premier ministre, ne semblent pas avoir été significativement enrayées.

When considered in a long-term perspective, Tony Blair's constitutional reforms appear to have been as much inspired by a desire to redress some of the alleged excesses perpetrated by the Tories during their eighteen years in power, as by the will to thoroughly modernise Britain's institutional framework. However, certain fundamental trends of recent decades, such as increasing centralisation and a greater concentration of power in the hands of the Prime Minister, do not seem to have been effectively reversed.

\section{AUTEUR}

\section{PHILIPPE CHASSAIGNE}

Université de Tours 